\title{
Intervenções psicopedagógicas e Discalculia do Desenvolvimento: uma Revisão Sistemática da Literatura
}

\author{
Psychopedagogical interventions and Developmental Dyscalculia: a \\ Systematic Review of the Literature
}

Intervenciones psicopedagógicas y Discalculia del Desarrollo: una Revisión Sistemática de la Literatura

\author{
* Lanúzia Almeida Brum Avila \\ Doutoranda pela Universidade Católica do Rio Grande do Sul, Porto Alegre, Rio Grande do Sul, Brasil. \\ lanuzia.avila@acad.pucrs.br

\section{** Isabel Cristina Machado de Lara} \\ Professora doutora na Universidade Católica do Rio Grande do Sul, Porto Alegre, Rio Grande do Sul, Brasil. \\ isabel.lara@pucrs.br
}

\section{*** Valderez Marina do Rosário Lima}

Professora doutora na Universidade Católica do Rio Grande do Sul, Porto Alegre, Rio Grande do Sul, Brasil. valderez.lima@pucrs.br

Recebido em 08 de março de 2019

Aprovado em 27 de julho de 2019

Publicado em 12 de setembro de 2019

\section{RESUMO}

Este artigo apresenta uma análise de teses, dissertações e artigos, no âmbito nacional, tendo como foco principal propostas de intervenções psicopedagógicas para estudantes do Ensino Fundamental com Discalculia do Desenvolvimento - DD, usando-se da Revisão Sistemática da Literatura - RSL, como método de pesquisa. Tem-se como questão guia de pesquisa "Como intervenções psicopedagógicas podem auxiliar estudantes do Ensino Fundamental com DD?". Desse modo, o objetivo é verificar a existência de propostas psicopedagógicas para serem desenvolvidas com esses estudantes, potencializando e reabilitando as habilidades matemáticas em defasagem na DD. Para selecionar as produções, utilizou-se da base de dados Biblioteca Digital Brasileira de Teses e Dissertações - BDTD e em relação à seleção dos artigos, optou-se pelo Google Acadêmico. Evidencia-se que o Brasil ainda carece de pesquisas na área da Educação Matemática que tenham como preocupação a avaliação, pois a falta de instrumentos padronizados impede que os profissionais de fato compreendam especificamente as áreas debilitadas e estabeleçam um plano de intervenção eficaz para os estudantes em tratamento terapêutico. Palavras-chave: Discalculia do Desenvolvimento; Habilidades matemáticas; Intervenções psicopedagógicas. 


\section{ABSTRACT}

This article presents an analysis of theses, dissertations and articles, at the national level, focusing mainly on proposals for psychopedagogical interventions for primary school students with Developmental Discalculia - DD, using the Systematic Review of Literature RSL, as a method of search. It has as a question guide research "How psychopedagogical interventions can help elementary school students with DD?" Thus, the objective is to verify the existence of psychopedagogical proposals to be developed with these students, potentializing and rehabilitating the mathematical abilities in delay in DD. In order to select the productions, the Brazilian Digital Library of Theses and Dissertations (BDTD) database was used and in relation to the selection of articles, we chose Google Scholar. It is evident that Brazil still lacks research in Mathematics Education that is concerned with evaluation, since the lack of standardized instruments prevents professionals from actually understanding the weak areas and establishing an effective intervention plan for students in therapeutic treatment.

Keywords: Developmental Dyscalculia; Mathematical skills; Psychopedagogical interventions.

\section{RESUMEN}

Este artículo presenta un análisis de tesis, disertaciones y artículos, en el ámbito nacional, teniendo como foco principal propuestas de intervenciones psicopedagógicas para estudiantes de la Enseñanza Fundamental con Discalculia del Desarrollo - DD, usando la Revisión Sistemática de la Literatura - RSL, como método de la investigación. Se tiene como cuestión guía de investigación "Cómo intervenciones psicopedagógicas pueden auxiliar a estudiantes de la Enseñanza Fundamental con DD?". De este modo, el objetivo es verificar la existencia de propuestas psicopedagógicas para ser desarrolladas con esos estudiantes, potenciando y rehabilitando las habilidades matemáticas en desfase en la DD. Para seleccionar las producciones, se utilizó la base de datos Biblioteca Digital Brasileña de Tesis y Disertaciones - BDTD y en relación a la selección de los artículos, se optó por Google Académico. Se evidencia que Brasil todavía carece de investigaciones en el área de la Educación Matemática que tengan como preocupación la evaluación, pues la falta de instrumentos estandarizados impide que los profesionales de hecho comprendan específicamente las áreas debilitadas y establezcan un plan de intervención eficaz para los estudiantes en tratamiento terapéutico.

Palabras clave: Discalculia del Desarrollo; Habilidades matemáticas; Intervenciones psicopedagógicas.

\section{Contextualização da pesquisa}

Na década de 1940, a Discalculia do Desenvolvimento - DD - foi definida por Gerstmann (1957) como sendo uma inabilidade em relação às operações simples e/ou complexas, dificuldade em compreender a orientação da sequência dos numerais e quanto às frações. Outras definições foram corroborando a definição do autor, como a dada por 
Bakwin (1960 apud KOSC, 1974), em que o autor destaca que estudantes com DD tendem a apresentar dificuldade em contar. Posteriormente, Cohn (1961 apud MYKLEBUST; JHONSON, 1962) e Gerstmann (1940 apud MYKLEBUST; JHONSON, 1962), mencionaram que a DD é ocasionada por uma disfunção cerebral, a qual interfere no reconhecimento e manipulação de numerais.

Considerando as definições existentes e com o propósito de sistematizar uma definição de DD, Myklebust e Jhonson (1962) salientam que o transtorno se manifesta como uma inabilidade em tarefas como a leitura e/ou escrita de numerais e dificuldade na compreensão dos símbolos matemáticos, embora a inteligência esteja dentro do esperado para a faixa etária.

Diante às definições existentes, Kosc (1970 apud KOSC, 1974) conceitua DD como sendo uma disfunção estrutural de habilidades matemáticas que está relacionada a uma deficiência genética ou congênita, sem apresentar outras disfunções que justifiquem as defasagens cognitivas.

Além de definir DD, Kosc (1974) propôs uma classificação em seis categorias, no intuito de definir e classificar especificamente cada uma das habilidades em defasagem. A seguir apresentam-se as habilidades em prejuízo em cada categoria.

Na primeira categoria, o autor (1974) remete-se à Discalculia verbal, em que as dificuldades referem-se às habilidades verbais, relacionadas aos termos e símbolos matemáticos, em que o estudante apresenta dificuldade em nomear quantidades e numerais; reconhecer os símbolos matemáticos.

Em relação à Discalculia Practognóstica, de acordo com Kosc (1974) o estudante tende a apresentar dificuldade na manipulação de objetos ou figuras, em específico na enumeração e comparação de quantidades.

No que se refere à Discalculia Léxica, o autor (1974) menciona que os estudantes com essa categoria de Discalculia apresentam dificuldades nos cálculos matemáticos, especificamente em sinais, dígitos e numerais relacionados à leitura dos símbolos matemáticos.

Em se tratando da Discalculia Gráfica, conforme o autor (1974) os estudantes apresentam dificuldade em escrever os símbolos matemáticos. Um estudante com essa categoria de Discalculia pode apresentar também Disgrafia e Dislexia. 
Para Kosc (1974), a Discalculia Ideognóstica, pode ser considerada como uma dificuldade na compreensão de conceitos matemáticos e na execução de operações mentais, mesmo somas simples, pode ser uma tarefa extremamente difícil.

A sexta categoria definida pelo autor (1974), remete-se à Discalculia Operacional, na qual os estudantes apresentam dificuldades na resolução de operações, ocorrendo à troca de operações, como por exemplo, em um cálculo de adição realizar uma subtração, ou viceversa.

Baseando-se na definição de Kosc (1974), principalmente no que se refere às categorias de DD sistematizadas pelo autor, Avila e Lara (2017) reportam-se a DD como um Transtorno de Aprendizagem - TA que acomete as habilidades específicas, necessitando avaliação e tratamento psicopedagógico. Conforme as autoras,

[...] a falta de instrumentos de avaliação capazes de proporcionarem com precisão que tipo de habilidade está em defasagem e a escassez de literatura sobre esse transtorno, dificulta a atividade desse profissional impedindo-o, muitas vezes, de realizar intervenções que de fato reabilitem as habilidades debilitadas. (AVILA; LARA, 2017, p. 53).

Todavia, mesmo a DD sendo um transtorno grave, em que as diferentes habilidades matemáticas estão em defasagem, podendo variar seu grau de severidade de estudante para estudante, "[...] constatou-se que por meio de intervenções psicopedagógicas baseadas, em particular, na utilização de jogos específicos para as dificuldades particulares de cada criança, é possível oportunizar melhoras significativas no desempenho em Matemática." (AVILA, 2017, p. 223).

A partir dessas considerações, uma análise de teses, dissertações e artigos que abordam a temática intervenção psicopedagógica para estudantes com DD, no âmbito nacional, usando-se como método de pesquisa a Revisão Sistemática da Literatura - RSL - pode propiciar avanços em pesquisas na Educação Matemática. Sendo assim, tem-se como questão guia de pesquisa "Como intervenções psicopedagógicas podem auxiliar estudantes do Ensino Fundamental com DD?". Nessa perspectiva, tem-se como objetivo, verificar a existência de propostas psicopedagógicas para serem desenvolvidas com os estudantes, potencializando e reabilitando as habilidades matemáticas em defasagem na DD.

Para tanto, realizou-se uma busca ao banco de bases de dados disponibilizados pela Biblioteca Digital Brasileira de Teses e Dissertações - BDTD; Google Acadêmico, 
possibilitando a identificação de teses, dissertações e artigos, no âmbito nacional, sem delimitação de período.

Os critérios de seleção usados foram às palavras-chave: "intervenções psicopedagógicas"; "habilidades matemáticas"; defasagem; Discalculia; "Discalculia do Desenvolvimento".

Para realizar a análise das teses e dissertações, elencaram-se os seguintes aspectos: título da produção; nome do acadêmico; ano de conclusão; instituição; palavras-chave; objetivo geral; autores utilizados para definir DD; problema de pesquisa; interesses de pesquisa das acadêmicas; contextos de pesquisa; principais metodologias e pesquisa empregadas; principais resultados; contribuições para o avanço do tema na área de ensino de Matemática; principais referências.

No que concerne à análise dos artigos, priorizou-se os seguintes aspectos: título; autor (es); interesses de pesquisa dos autores; contribuições para o avanço do tema na área de ensino Matemática; principais referências.

\section{Etapas percorridas na Revisão Sistemática da Literatura}

Esta RSL foi realizada com base nos critérios propostos por Pickering e Bryrne (2014). Para os autores, a utilização desse método de pesquisa justifica-se quando o pesquisador necessita identificar a existência ou não de produções referentes ao tema investigado em sua área de atuação (PICKERING; BRYRNE, 2014). A seguir apresentam-se as etapas percorridas nesse estudo.

Primeiramente, definiu-se o tema principal deste estudo, que consiste em uma revisão de pesquisas acadêmicas sobre intervenções psicopedagógicas para estudantes do Ensino Fundamental com DD, no âmbito nacional (fase 1).

Posteriormente, formulou-se a questão guia de pesquisa: "Como intervenções psicopedagógicas podem auxiliar estudantes do Ensino Fundamental com DD?" (fase 2).

Em seguida, determinou-se as palavras-chave para realizar a busca em duas bases de dados. As palavras-chave para a busca foram: "intervenções psicopedagógicas", "habilidades matemáticas", defasagem, Discalculia, "Discalculia do Desenvolvimento". Todavia, convém mencionar que em cada uma das bases foram realizadas combinações específicas de palavras (fase 3 ).

Para realizar a busca, optou-se pelas bases BDTD e Google Acadêmico. A BDTD foi utilizada para busca de teses e dissertações, pois disponibiliza textos completos dos 
trabalhos acadêmicos nacionais de Instituições de Ensino Superior. O Google Acadêmico foi utilizado para busca de artigos nacionais, na intenção de ampliar a pesquisa, pois outras bases apresentavam um número reduzido de documentos de acordo com as palavraschave (fase 4).

Feito isso, com base no objetivo dessa pesquisa, foram estabelecidos critérios de inclusão e exclusão, para a seleção das produções e dos artigos. Em relação aos critérios de inclusão destacam-se: ser artigo de periódicos, tese ou dissertação; apresentar propostas de intervenções psicopedagógicas para estudantes do Ensino Fundamental com DD e estar relacionado à questão que guiou a RSL. Quanto aos critérios de exclusão, mencionam-se: referir-se as Dificuldades de Aprendizagem em Matemática - DAM; tratar de outros Transtornos de Aprendizagem - TA; incompatibilidade com a questão de pesquisa; estar relacionado ao Ensino Médio e/ou Ensino Superior (fase 5).

Na próxima etapa, realizou-se a leitura de cada uma das produções encontradas na BDTD, com o objetivo de selecionar o corpus de análise, deste estudo. A leitura das produções priorizou os títulos, resumos, introduções, procedimentos metodológicos das produções, análise dos resultados, dentre outros. E em específico, acerca dos artigos do Google Acadêmico, realizou-se a leitura dos resumos, palavras-chave e introdução, de cada um dos artigos (fase 6).

Feito isso, organizou-se dois bancos de dados no Excel, um para as produções acadêmicas da BDTD e outro para os artigos do Google Acadêmico, inserindo, em ambos, o corpus de análise. Logo em seguida, considerando os critérios de inclusão, organizou-se as produções e os artigos em categorias (fase 7).

De posse do corpus para análise, realizou-se a leitura minuciosa das produções e dos artigos pré-selecionados, visando à questão que guiou essa revisão (fase 8 ).

Posteriormente, considerando os critérios de inclusão e exclusão delimitados, foram testadas e revisadas as categorias determinadas em cada uma das bases de dados (fase 9). Em seguida, organizou-se, no Excel, dois bancos de dados, com o corpus definitivo das produções e dos artigos (fase 10).

Realizada a organização dos bancos de dados, descreveu-se os métodos usados no desenvolvimento da RSL (fase 11). Feito isso, analisou-se cuidadosamente os resultados e as conclusões advindos da revisão (fase 12). Com base nas análises, das produções e dos artigos, iniciou-se a descrição dos resultados e a elaboração de discussões dos dados 
obtidos (fase 13). Logo, dedicou-se a elaboração da introdução, resumo e referências (fase 14).

E para finalizar, conforme orienta Pickering e Bryrne (2014), realizou-se a releitura do artigo, revisando-se cada uma das fases propostas pelos autores (fase 15).

A seguir apresentam-se com detalhes a busca em cada uma das bases e os resultados emergentes.

\section{Biblioteca Digital Brasileira de Teses e Dissertações}

Para realizar a busca de teses e dissertações no Brasil, sem período de tempo, foram utilizadas as informações da BDTD, por meio da busca de coleções on-line assinadas por uma universidade privada de Porto Alegre, disponíveis no Portal de Artigos da Capes e de acesso livre aos estudantes vinculados à universidade.

Ao acessar a página, e digitar as palavras no campo de busca, tem-se a opção buscar ou busca avançada. A busca pode ser refinada, selecionando as opções, instituições, repositório, programa, autor, contribuidor, orientador (a), tipo de documento, idioma, assunto, assunto em inglês, área de conhecimento, ano de defesa. Optou-se por buscar em todos os campos, sem delimitação de período.

Convém salientar, que ao realizar a busca das palavras-chaves, todas as palavras são procuradas em várias partes das produções: título, assunto, palavras-chave e resumo das teses e dissertações. Sendo assim, para uma busca mais detalhada, faz-se necessário a leitura dos resumos, das palavras-chave, da introdução e dos procedimentos metodológicos de todas as produções.

Referente à busca realizada, primeiramente foram digitadas as palavras (Discalculia OR "Discalculia do Desenvolvimento"), obtendo-se sete teses e 20 dissertações. Posteriormente, buscou-se pelas palavras ("intervenções psicopedagógicas") AND (Discalculia OR "Discalculia do Desenvolvimento"), obtendo-se duas dissertações. A busca foi finalizada, utilizando-se as palavras ("intervenções psicopedagógicas") AND ("habilidades matemáticas") AND (defasagem) AND (Discalculia OR "Discalculia do Desenvolvimento"), obtendo-se uma dissertação.

Para apresentar os resultados encontrados nessa busca, elaborou-se o Quadro 1. 


\section{Educação Especial}

http://dx.doi.org/10.5902/1984686X37223

Quadro 1 - Resultado da busca com as palavras-chave

\begin{tabular}{|c|c|c|c|}
\hline BASE DE DADOS & PALAVRAS-CHAVE & TESES & DISSERTAÇŌES \\
\hline \multirow{3}{*}{ BDTD } & $\begin{array}{l}\text { (Discalculia OR “Discalculia do } \\
\text { Desenvolvimento") }\end{array}$ & 7 & 22 \\
\hline & $\begin{array}{l}\text { ("intervenções } \\
\text { psicopedagógicas") AND } \\
\text { (Discalculia OR "Discalculia do } \\
\text { Desenvolvimento") }\end{array}$ & 0 & 2 \\
\hline & $\begin{array}{l}\text { ("intervenções } \\
\text { psicopedagógicas") AND } \\
\text { ("habilidades matemáticas") } \\
\text { AND (defasagem) AND } \\
\text { (Discalculia OR "Discalculia do } \\
\text { Desenvolvimento") }\end{array}$ & 0 & 1 \\
\hline
\end{tabular}

Fonte: Dados organizados pelas autoras a partir de consulta a BDTD.

No Quadro 1, é possível perceber que conforme foram sendo acrescidas novas palavras-chave, na intenção de buscar estudos que contemplassem o tema principal deste estudo, foi diminuindo a quantidade de produções, de 20 teses e dissertações para uma dissertação, a qual remete-se ao estudo de Avila (2017).

$\mathrm{Na}$ intenção de apresentar com detalhes a busca realizada na BDTD, sem delimitação do período, apresenta-se a frequência anual das produções acadêmicas usando-se as palavras-chave (Discalculia OR “Discalculia do Desenvolvimento”), por meio do Gráfico 1.

Gráfico 1 - Relação das produções acadêmicas no Brasil no período de 1991 a 2017

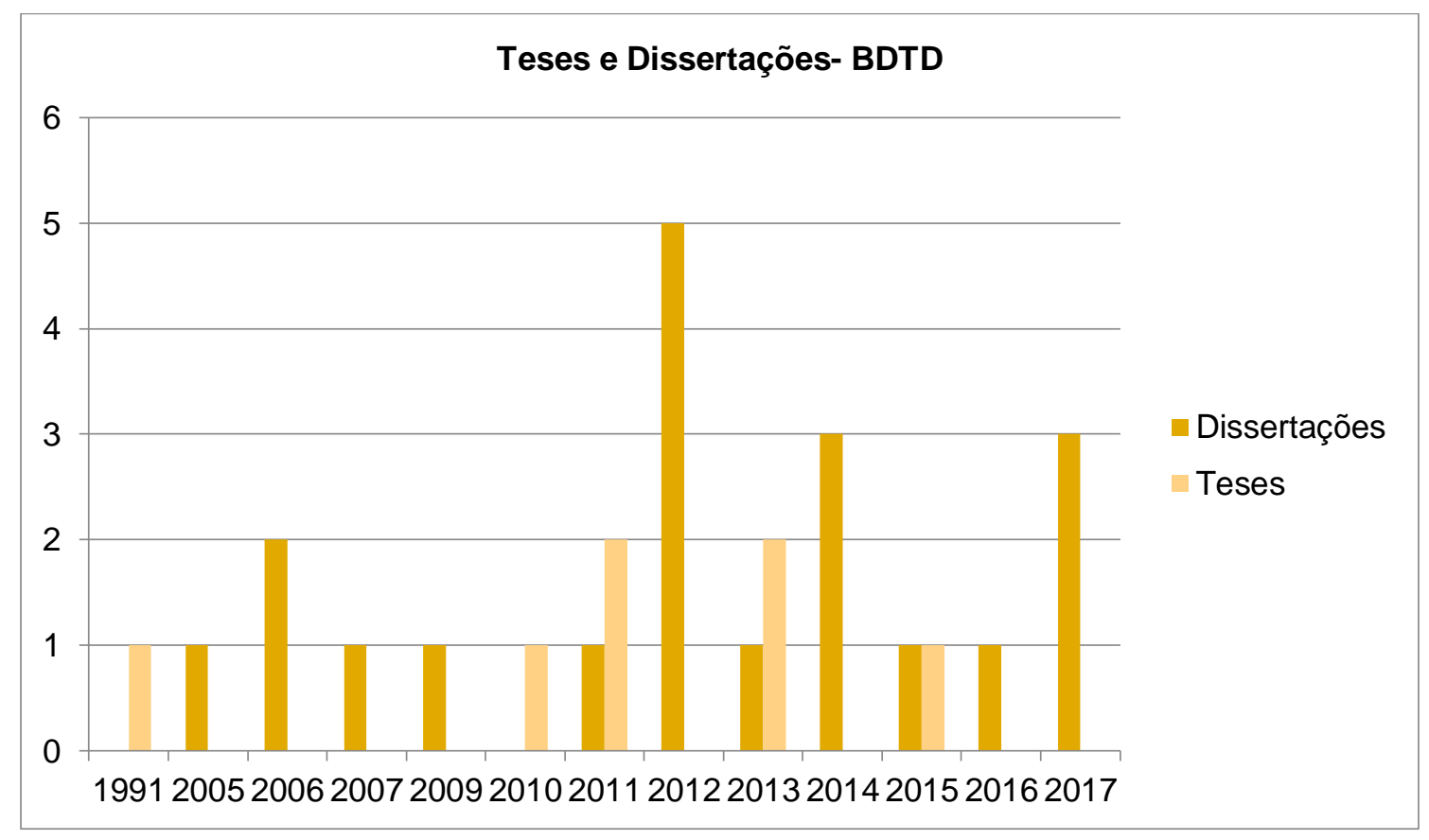

Fonte: Elaborada pelas autoras a partir de consulta a BDTD.

Analisando o Gráfico 1, percebe-se que a maioria das produções acadêmicas de mestrado foram defendidas com mais frequência no período de 2012 a 2017. Sendo assim, 
percebe-se que o número de pesquisadores interessados no estudo da DD tem aumentado, porém ainda se faz necessário avançar nas pesquisas no meio médico e educacional acerca do assunto.

Foi possível verificar que ambas as dissertações que apareceram na $2^{a}$ busca, usando-se das palavras-chaves ("intervenções psicopedagógicas") AND (Discalculia OR "Discalculia do Desenvolvimento") e uma dissertação na $3^{a}$ busca, utilizando-se das palavras-chaves ("habilidades matemáticas") AND (defasagem) AND (Discalculia OR "Discalculia do Desenvolvimento"), são as mesmas inseridas na $1^{\text {a }}$ busca.

$\mathrm{Na}$ intenção de apresentar as 9 categorias organizadas, após a leitura dos resumos, palavras-chave e conclusões das produções, apresenta-se o Quadro 2.

Quadro 2 - Categorias das produções

\begin{tabular}{|c|c|}
\hline CATEGORIAS & NÚMERO DE PRODUÇÕES \\
\hline Estudos sobre aprendizagem Matemática & 6 \\
\hline Avaliação e intervenções pedagógicas & 4 \\
\hline Avaliação das habilidades matemáticas & 3 \\
\hline Dificuldades de Aprendizagem & 2 \\
\hline Educação especial & 5 \\
\hline Genética e aprendizagem & 3 \\
\hline Intervenções neuropsicológicas & 2 \\
\hline Intervenções psicopedagógicas & 2 \\
\hline Transtornos de Aprendizagem & 2 \\
\hline
\end{tabular}

Fonte: Elaborada pela autora a partir de consulta a BDTD

Realizou-se a leitura minuciosa dos resumos, palavras-chave e conclusões das produções, e se selecionou para a leitura integral na BDTD somente duas dissertações da categoria intervenções psicopedagógicas, pois contemplavam os critérios de inclusão.

Com base na leitura das produções, percebeu-se que ainda há poucos estudos tratando da avaliação psicopedagógica, ocasionando assim, falta de instrumentos capazes de proporcionarem com precisão que tipo de habilidade está em defasagem e a escassez de literatura sobre esse transtorno dificulta a atividade do psicopedagogo, impedindo-o, muitas vezes, de realizar intervenções que de fato reabilitem as habilidades debilitadas, conforme aponta Avila (2017).

\section{Artigos selecionados no Google Acadêmico}

A busca de artigos nacionais, sem delimitação de período, ocorreu por meio do Google acadêmico no site http://scholar.google.com.br, sem exigência de login, sendo de livre ascesso para todos os interessados em pesquisar informações científicas.

Ao acessar a página, é possível optar entre pesquisar em qualquer idioma ou pesquisar páginas em português, além da escolha entre pesquisa simples ou pesquisa 
avançada. É possível, refinar a busca pelas opções: a qualquer momento ou período específico, classificar por relevância, incluir patentes e incluir citações. Optou-se pela pesquisa simples, pesquisar páginas em português, sem delimitação de período específico.

Porém, ao realizar a busca das palavras-chave, todas as palavras são procuradas nas produções acadêmicas, bem como: artigos, relatórios, trabalhos de conclusão, documentos de eventos, dissertações, teses, livros, dentre outros. Para realizar a busca, é necessário realizar a leitura de todos os títulos dos documentos e acessá-los, de modo, a saber, o tipo de documento. Nesse caso, em específico, como se optou em buscar artigos, ao encontrálos, para uma busca detalhada, realizou-se a leitura dos resumos, palavras-chave e introdução, de cada um dos artigos.

Em se tratando da busca realizada no Google Acadêmico, primeiramente foram digitadas as palavras "(Discalculia OR Discalculia do Desenvolvimento)", obtendo-se 37 artigos. Posteriormente, buscou-se pelas palavras ("intervenções psicopedagógicas") AND (Discalculia OR "Discalculia do Desenvolvimento"), obtendo-se nove artigos. A busca foi finalizada, utilizando-se as palavras ("intervenções psicopedagógicas") AND ("habilidades matemáticas") AND (defasagem) AND (Discalculia OR "Discalculia do Desenvolvimento") obtendo-se um artigo.

Para apresentar os resultados encontrados nessa busca, elaborou-se o Quadro 3.

Quadro 3 - Resultado da busca avançada com as palavras-chave

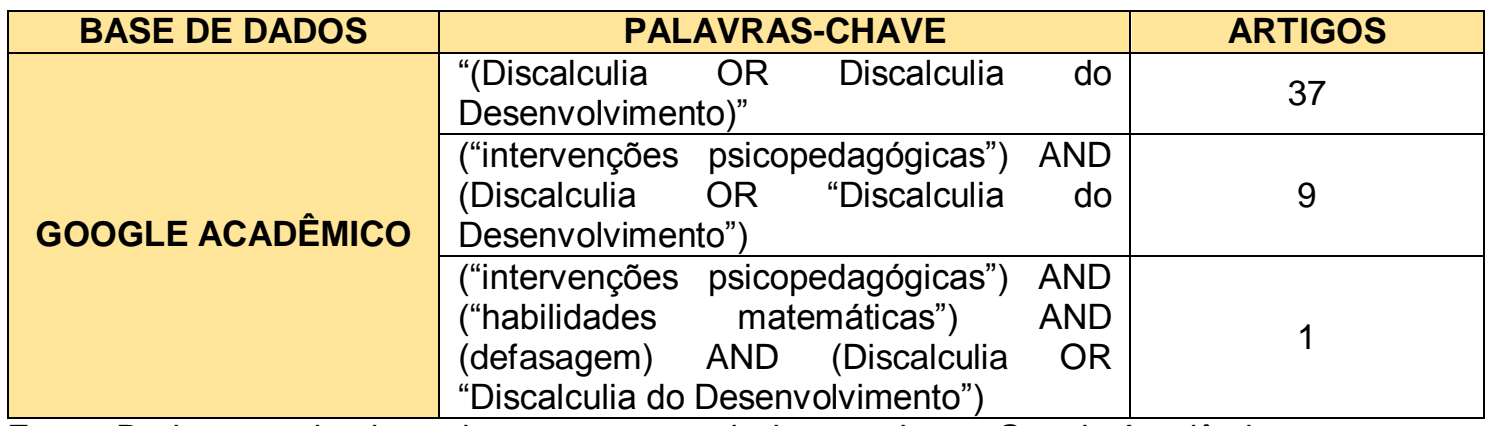

Fonte: Dados organizados pelas autoras a partir de consulta ao Google Acadêmico.

Considerando o Quadro 3, percebe-se a cada busca realizada, conforme foram acrescidas novas palavras-chave, com o objetivo de responder à questão guia de pesquisa deste estudo foi reduzindo a quantidade de artigos de 37 para um artigo, o qual remete-se ao artigo "Discalculia: Um mapeamento de artigos brasileiros" de Avila e Lara (2017). Todavia o objetivo das autoras, em específico neste artigo, foi verificar o estado da arte das produções acadêmicas relacionadas às DAM e ao TAM, buscando por suas convergências e divergências em relação a alguns aspectos, entre eles o modo como são conceituados 
os termos DD, TAM e DAM e as contribuições aos profissionais da área da Educação Matemática. O artigo foi desenvolvido paralelamente a produção da dissertação "Avaliação e intervenções psicopedagógicas em crianças com indícios de Discalculia", a qual já foi mencionada nessa revisão, fazendo com que na busca pelas palavras-chave ("intervenções psicopedagógicas") AND ("habilidades matemáticas") AND (defasagem) AND (Discalculia OR "Discalculia do Desenvolvimento"), fosse rastreado.

Os detalhes das três buscas realizadas no Google Acadêmico, sem delimitação do período, podem ser observados a seguir no Gráfico 2.

Gráfico 2 - Frequência dos artigos no Brasil no período de 2008 a 2018

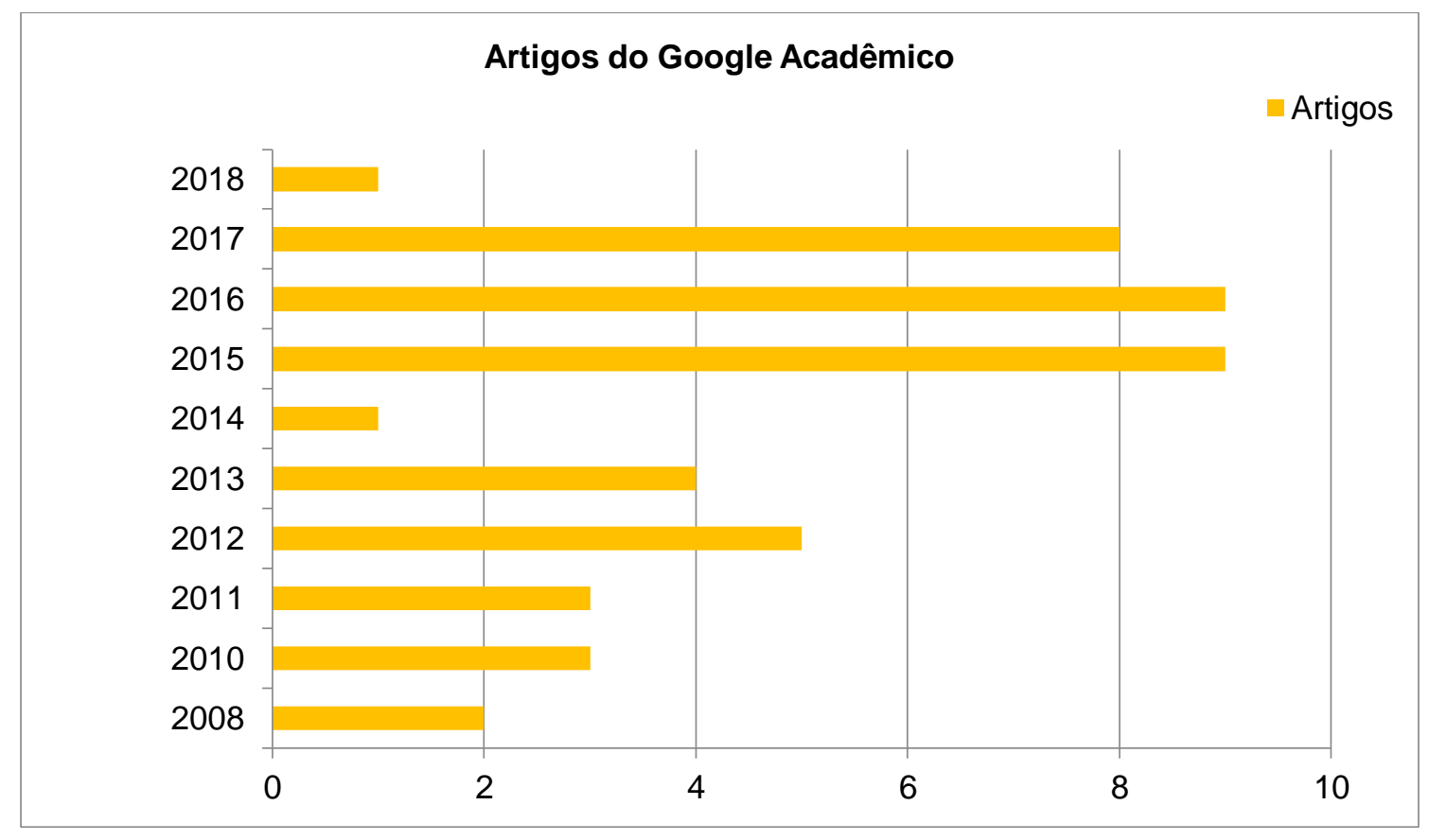

Fonte: Elaborada pelas autoras a partir de consulta no Google Acadêmico.

Observando a frequência dos artigos no Gráfico 2, pode-se observar no período de 2008 a 2018 um aclive no número de artigos produzidos sobre DD, porém a frequência acentuada de produções ocorreu entre 2015 e 2017.

Nas buscas realizadas, verificou-se que dois artigos repetem-se duas vezes. Quando utilizado "(Discalculia OR Discalculia do Desenvolvimento)" e posteriormente, as palavras ("intervenções psicopedagógicas") AND (Discalculia OR “Discalculia do Desenvolvimento"), a produção dos autores Bernardi e Stobaus (2016) aparece em ambas as buscas. O artigo de Avila e Lara (2017) está contemplado nas buscas pelas palavras-chave ("intervenções psicopedagógicas") AND (Discalculia OR "Discalculia do Desenvolvimento") e após, em 
("intervenções psicopedagógicas") AND (“habilidades matemáticas”) AND (defasagem) AND (Discalculia OR "Discalculia do Desenvolvimento").

A partir da leitura dos resumos, palavras-chave e conclusões das produções foi possível organizar essas pesquisas em 11 categorias, conforme o Quadro 4.

Quadro 4 - Categorias das produções

\begin{tabular}{|c|c|}
\hline CATEGORIAS & NÚMERO DE PRODUÇÕES \\
\hline Estudos sobre aspectos neurobiológicos & 4 \\
\hline Concepções acerca da DD & 4 \\
\hline Cognição numérica & 7 \\
\hline Déficit de Atenção e Hiperatividade & 1 \\
\hline Dificuldades e Transtornos de Aprendizagem & 7 \\
\hline Dificuldades de Aprendizagem em Matemática & 6 \\
\hline Intervenções neuropsicológicas & 2 \\
\hline Intervenções psicopedagógicas & 2 \\
\hline Intervenções psicopedagógicas no Ensino Superior & 1 \\
\hline Memória de trabalho & 1 \\
\hline Estudos sobre aprendizagem Matemática & 8 \\
\hline
\end{tabular}

Fonte: Elaborada pelas autoras a partir de consulta no Google Acadêmico.

Considerando o propósito da RSL, realizou-se a leitura minuciosa dos resumos, palavras-chave e conclusões das produções e se selecionou para a leitura integral, no Google Acadêmico, dois artigos da categoria intervenções psicopedagógicas.

\section{Resultados/discussão}

\section{Produções acadêmicas selecionadas na biblioteca digital brasileira de teses e}

\section{dissertações}

Elaborou-se o Quadro 5, no qual consta o ano da defesa, o título, o autor, o orientador e a IES, respectivamente, na intenção de apresentar as dissertações selecionadas para leitura integral.

Quadro 5 - Dissertações selecionadas para leitura integral e análise.

\begin{tabular}{|l|l|l|l|l|l|}
\hline CÓDIGO & $\begin{array}{c}\text { ANO } \\
\text { DA } \\
\text { DEF } \\
\text { ESA }\end{array}$ & \multicolumn{1}{|c|}{ TíTULO } & $\begin{array}{l}\text { AUTOR } \\
\text { (A) }\end{array}$ & $\begin{array}{l}\text { ORIENTADOR } \\
\text { (A) }\end{array}$ & \multicolumn{1}{|c|}{ IES } \\
\hline D11 & 2017 & $\begin{array}{l}\text { Avaliação e intervenções } \\
\text { psicopedagógicas em crianças } \\
\text { com indícios de Discalculia }\end{array}$ & $\begin{array}{l}\text { Lanúzia } \\
\text { Almeida } \\
\text { Brum } \\
\text { Avila }\end{array}$ & $\begin{array}{l}\text { Isabel Cristina } \\
\text { Machado de } \\
\text { Lara }\end{array}$ & $\begin{array}{l}\text { Pontifícia Universidade } \\
\text { Católica do Rio Grande do } \\
\text { Sul }\end{array}$ \\
\hline D2 & 2006 & $\begin{array}{l}\text { Alunos com discalculia: o } \\
\text { resgate da auto-estima e da da } \\
\text { auto-imagem através do lúdico }\end{array}$ & $\begin{array}{l}\text { Jussara } \\
\text { Bernard } \\
\text { es }\end{array}$ & $\begin{array}{l}\text { Claus Dieter } \\
\text { Stobäus }\end{array}$ & $\begin{array}{l}\text { Pontífícia Universidade } \\
\text { Católica do Rio Grande do } \\
\text { Sul }\end{array}$ \\
\hline
\end{tabular}

Fonte: Elaborada pelas autoras a partir dos dados disponíveis na BDTD.

${ }^{1}$ Para representar cada produção utilizou-se Dn (D1, D2, ...) para Dissertações. 
Apresenta-se uma síntese detalhada dos aspectos relevantes das dissertações no Quadro 6, considerando os seguintes aspectos: título da produção, nome do acadêmico, ano de conclusão, instituição, palavras-chave, objetivo geral, autores utilizados para definir DD, problema de pesquisa; interesses de pesquisa da acadêmica; contextos de pesquisa; principais metodologias e pesquisa empregadas; principais resultados; contribuições para o avanço do tema na área de ensino de Matemática e principais referências.

Quadro 6 - Síntese das dissertações selecionadas

\begin{tabular}{|c|c|}
\hline IDENTIFICADOR & D1 \\
\hline \multicolumn{2}{|c|}{$\begin{array}{c}\text { Avaliação e intervenções psicopedagógicas em crianças com indícios de Discalculia. } \\
\text { Acadêmica: Lanúzia Almeida Brum Avila }\end{array}$} \\
\hline ANO/IES & 2017/ PUCRS \\
\hline PALAVRAS-CHAVE & $\begin{array}{l}\text { Discalculia. Diagnóstico. Intervenções psicopedagógicas. Habilidades } \\
\text { matemáticas. }\end{array}$ \\
\hline OBJETIVO GERAL & $\begin{array}{l}\text { Analisar a evolução do desenvolvimento das habilidades matemáticas } \\
\text { envolvidas na DD de crianças com indícios desse transtorno, após a } \\
\text { realização de intervenções psicopedagógicas. }\end{array}$ \\
\hline $\begin{array}{l}\text { AUTORES UTILIZADOS PARA } \\
\text { DEFINIR DISCALCULIA }\end{array}$ & $\begin{array}{l}\text { Bastos (2006); Butterworth (2005); Dehaene (1995); Fonseca (1995); } \\
\text { García (1998); Haase, Costa, Michelli, Oliveira, Wood (2011), Kosc } \\
\text { (1974), Lara (2004); Rotta (2006); Vieira (2004). }\end{array}$ \\
\hline PROBLEMA DE PESQUISA & $\begin{array}{l}\text { Como intervenções psicopedagógicas repercutiram na evolução do } \\
\text { desenvolvimento das habilidades matemáticas envolvidas na DD em } \\
\text { crianças com indícios desse transtorno? }\end{array}$ \\
\hline INTERESSES DE PESQUISA & $\begin{array}{l}\text { Considerando sua trajetória profissional e consciente dos problemas } \\
\text { decorrentes da DD no desenvolvimento acadêmico e emocional das } \\
\text { crianças, a pesquisadora buscou priorizar a avaliação e propostas de } \\
\text { intervenções psicopedagógicas para crianças com indícios de DD, } \\
\text { pois no Brasil ainda carecemos de pesquisas na Educação que } \\
\text { tenham esse foco, o que dificulta o trabalho dos psicopedagogos, pela } \\
\text { falta de instrumentos para avaliar que consequentemente interfere no } \\
\text { processo de intervenção. }\end{array}$ \\
\hline CONTEXTO DE PESQUISA & $\begin{array}{l}\text { As testagens de QI foram realizadas no ambulatório do Hospital São } \\
\text { Lucas - PUCRS de Porto Alegre. As testagens e intervenções com o } \\
\text { grupo experimental ocorreram no Laboratório de aprendizagem de } \\
\text { Matemática da Escola de Ciências da PUCRS. E o processo de } \\
\text { avaliação e intervenção do grupo controle, foram desenvolvidos na } \\
\text { sala de recursos de uma Escola Estadual do Município de Capivari do } \\
\text { Sul. }\end{array}$ \\
\hline
\end{tabular}




\begin{tabular}{|c|c|}
\hline $\begin{array}{l}\text { PRINCIPAIS METODOLOGIAS } \\
\text { E PESQUISA EMPREGADAS }\end{array}$ & $\begin{array}{l}\text { A partir da análise de D1, verifica-se que a autora optou pelo método } \\
\text { de pesquisa de abordagem qualitativa e quantitativa de delineamento } \\
\text { experimental. } \\
\text { Instrumentos para coleta de dados: 1ํ) Avaliação - Teste de } \\
\text { Transcodificação (MOURA; MADEIRA; CHAGAS; LONNEMANN; } \\
\text { KRINZINGER; WILLMES; HAASE, 2013); Subteste de Aritmética } \\
\text { (STEIN, 1994); Escala Wechsler Abreviada de Inteligência - WASI } \\
\text { (2014). } \\
\left.2^{\circ}\right) \text { Intervenção psicopedagógicas - Foram elaboradas as } \\
\text { intervenções psicopedagógicas, distribuídas em cinco aulas de } 2 \\
\text { horas para os atendimentos com o grupo controle e } 10 \text { sessões de } 1 \\
\text { hora para os atendimentos com o grupo experimental. Para o grupo } \\
\text { controle, foram realizadas atividades em grupo seguindo um } \\
\text { planejamento mais próximo do realizado em aula. Já, para o grupo } \\
\text { experimental, as intervenções foram planejadas com jogos } \\
\text { diferenciados para cada criança, levando-se em conta suas } \\
\text { habilidades matemáticas em defasagem e as habilidades que } \\
\text { necessitavam ser reabilitadas, considerando cada uma das } \\
\text { categorias de DD definidas por Kosc (1974). } \\
3^{\circ} \text { ) Reavaliação psicopedagógica. }\end{array}$ \\
\hline PRINCIPAIS RESULTADOS & $\begin{array}{l}\text { Por meio do trabalho realizado pela pesquisadora, é perceptível que } \\
\text { os jogos desenvolvidos, possibilitaram avanços no desempenho em } \\
\text { Matemática das crianças participantes do estudo, evidenciando-se } \\
\text { assim, que é possível por meio do lúdico, trabalhar as dificuldades } \\
\text { individuais, potencializando as habilidades preservadas e reabilitando } \\
\text { as habilidades em defasagem. }\end{array}$ \\
\hline CONTRIBUIÇÕES & $\begin{array}{l}\text { Os jogos utilizados nos atendimentos foram eficazes para o } \\
\text { tratamento de crianças com indícios de DD, contribuindo para concluir } \\
\text { que as habilidades matemáticas em defasagem podem ser } \\
\text { reabilitadas, potencializando as existentes. }\end{array}$ \\
\hline PRINCIPAIS REFERÊNCIAS & $\begin{array}{l}\text { BASTOS, J. A. Discalculia: transtorno específico da habilidade em } \\
\text { matemática. In: ROTTA, N.; OHLWEILER, L.; RIESCO, R. (Orgs). } \\
\text { Transtornos da aprendizagem: Abordagem neurobiológica e } \\
\text { multidisciplinar. São Paulo: Artes médicas, 2006, p. 195- } 206 \text {. } \\
\text { BUTTERWORTH, B. Developmental Dyscalculia. In: DEHAENE, S.; } \\
\text { COHEN, L. Towards an Anatomical and Functional Model of Number } \\
\text { Processing. Mathematical Cognition, London, v. 1, p. 83-120, } 1995 . \\
\text { FONSECA, V. Introdução às Dificuldades de Aprendizagem. } 2 \text {. ed. } \\
\text { Porto Alegre: Artes médicas, } 1995 \text {. } \\
\text { GARCÍA, J. N. Manual de dificuldades de aprendizagem: } \\
\text { Linguagem, leitura, escrita e matemática. Porto Alegre: Artes } \\
\text { Médicas, 1998. } \\
\text { HAASE, V. G.; COSTA, D. S.; MICHELLI, L. R.; OLIVEIRA, L. F. S.; } \\
\text { WOOD, G. O estatuto nosológico da discalculia do desenvolvimento. } \\
\text { In: CAPOVILLA, F. C. (Org). Transtornos de aprendizagem 2: Da } \\
\text { análise laboratorial e da reabilitação clínica para as políticas públicas } \\
\text { de prevenção pela via da educação, Memnon Edições Científicas: } \\
\text { São Paulo, 2011, p. 139-144. }\end{array}$ \\
\hline
\end{tabular}




\begin{tabular}{|c|c|}
\hline PRINCIPAIS REFERÊNCIAS & $\begin{array}{l}\text { KOSC, L. Developmentol Dyscalculia. Journal of Learning } \\
\text { Disabilities can be found at, v. 7, n. 3, p.163-177, marc. } 1974 . \\
\text { LARA, I. C. M. Ensino inadequado de Matemática. Revista } \\
\text { Ciências e Letras, n. 35, p. 137-152, mar./jul. 2004. } \\
\text { ROTTA, N. T.; OHLWEILER, L., RIESCO, R. S. (Orgs). } \\
\text { Transtornos da aprendizagem: Abordagem neurobiológica e } \\
\text { multidisciplinar. São Paulo: Artes médicas, } 2006 \text {. } \\
\text { VIEIRA, Elaine. Transtornos na aprendizagem da matemática: } \\
\text { Número e discalculia. Revista Ciências e Letras, n. 35, p. 109-120, } \\
\text { mar./jul. 2004. }\end{array}$ \\
\hline IDENTIFICADOR & D2 \\
\hline \multicolumn{2}{|c|}{$\begin{array}{c}\text { Alunos com Discalculia: o resgate da autoestima e da autoimagem através do lúdico. Acadêmica: } \\
\text { Jussara Bernardes }\end{array}$} \\
\hline ANO/IES & 2006/ PUCRS \\
\hline PALAVRAS-CHAVE & Discalculia. autoimagem e autoestima. lúdico. \\
\hline OBJETIVO GERAL & $\begin{array}{l}\text { Verificar a influência do lúdico na autoestima e na autoimagem de } \\
\text { cinco crianças com DD que estão em atendimento psicopedagógico } \\
\text { no Laboratório de Aprendizagem, através da intervenção educativa } \\
\text { baseada na ludicidade. }\end{array}$ \\
\hline $\begin{array}{l}\text { AUTORES UTILIZADOS PARA } \\
\text { DEFINIR DISCALCULIA }\end{array}$ & $\begin{array}{l}\text { Benton (1987); Fonseca (1995); Garcia (1998); Kosc (1974); Lara } \\
\text { (2004); Vieira (2004). }\end{array}$ \\
\hline PROBLEMA DE PESQUISA & $\begin{array}{l}\text { Como o lúdico pode influenciar nos níveis de autoestima e de } \\
\text { autoimagem em crianças com DD? }\end{array}$ \\
\hline INTERESSES DE PESQUISA & $\begin{array}{l}\text { O percurso como professora do laboratório de aprendizagem e o } \\
\text { trabalho diário com os alunos com DAM suscitou na acadêmica, } \\
\text { questionamentos e reflexões sobre as dificuldades vivenciadas por } \\
\text { muitas crianças no âmbito escolar, no intuito de possibilitar aos } \\
\text { professores meios para que desenvolvam um trabalho que } \\
\text { contemple os diferentes estilos de aprendizagem, possibilitando que } \\
\text { as crianças com dificuldades de aprendizagem em matemática } \\
\text { possam aprender. }\end{array}$ \\
\hline CONTEXTO DE PESQUISA & $\begin{array}{l}\text { Os espaços escolares abrangidos pela investigação foram uma sala } \\
\text { de aula e o laboratório de aprendizagem de uma escola de Ensino } \\
\text { Fundamental no município de Porto Alegre. }\end{array}$ \\
\hline $\begin{array}{l}\text { PRINCIPAIS METODOLOGIAS } \\
\text { E PESQUISA EMPREGADAS }\end{array}$ & $\begin{array}{l}\text { A pesquisadora em D2 optou pela pesquisa, de cunho qualitativo- } \\
\text { quantitativo e caracterizou-se como estudo de caso. Instrumentos } \\
\text { para a coleta de dados: Teste Neuropsicológico Infantil, conhecido } \\
\text { como a Bateria de Luria - DNI; Questionário de autoestima e } \\
\text { autoimagem, entrevista semiestruturada com pais e professora; } \\
\text { observações descritivas e diário de campo, para o registro das } \\
\text { intervenções psicopedagógicas. }\end{array}$ \\
\hline PRINCIPAIS RESULTADOS & $\begin{array}{l}\text { Considerando a análise realizada, observa-se que o trabalho com o } \\
\text { lúdico influenciou positivamente a autoestima e a autoimagem das } \\
\text { crianças participantes da pesquisa. Constatou-se ainda avanços em } \\
\text { relação à aprendizagem da Matemática, a partir do emprego do } \\
\text { lúdico nos atendimentos desenvolvidos no laboratório de } \\
\text { aprendizagem. }\end{array}$ \\
\hline CONTRIBUIÇÕES & $\begin{array}{l}\text { O estudo desenvolvido pela pesquisadora buscou priorizar o } \\
\text { atendimento educacional inclusivo, conscientizando os professores } \\
\text { da necessidade da escola ser um espaço preparado para atender } \\
\text { as necessidades educativas específicas dos estudantes, } \\
\text { potencializando o seu desenvolvimento integral, impedindo a } \\
\text { repetência, o fracasso, a evasão e, consequentemente, a exclusão } \\
\text { social. }\end{array}$ \\
\hline
\end{tabular}


Conclusão

\begin{tabular}{|c|c|}
\hline PRINCIPAIS REFERÊNCIAS & $\begin{array}{l}\text { BENTON, A. F. L. Mathematical disability and the Gerstmann } \\
\text { Syndrome. In: DELOCHE, G.; SERON, X. Mathematical } \\
\text { disabilities: a cognitive neuropsychological perspective. } \\
\text { Lawrence Erlbaum Associates. Hillsdale, New Jersey: 1987, } \\
\text { p. } 111-120 \text {. } \\
\text { FONSECA, Vítor. Introdução às dificuldades de } \\
\text { aprendizagem. 2. ed. Porto Alegre: Artes Médicas, 1995. } \\
\text { GARCIA, José N. Manual de dificuldades de } \\
\text { aprendizagem: linguagem, leitura, escrita e matemática. } \\
\text { Porto Alegre: Artes Médicas, 1998. } \\
\text { KOSC, Ladislav. Developmental dyscalculia. Journal of } \\
\text { Learning Disabilities, v. 7, p. 164177, 1974. } \\
\text { LARA, Isabel C. M. Ensino inadequado de matemática. } \\
\text { Revista Ciências e Letras, n. } 35 \text {, p. 137-152, } 2004 \text {. } \\
\text { VIEIRA, Elaine. Transtornos na aprendizagem da } \\
\text { matemática: número e discalculia. Revista Ciências e } \\
\text { Letras, n. 35, p. 109-119, } 2004 \text {. }\end{array}$ \\
\hline
\end{tabular}

Fonte: Elaborada pelas autoras.

Após a realização da leitura detalhada de cada uma das dissertações selecionadas, foi possível perceber que a escolha pelo tema DD, está centrada na formação acadêmica e na experiência vivenciada por cada uma das acadêmicas, considerando a consciência da problemática acerca das DAM e do TAM, presentes em muitas escolas e da necessidade de um trabalho especializado nos laboratórios de aprendizagem e/ou consultórios psicopedagógicos.

Em relação ao objetivo geral, observa-se que as produções por um lado diferenciamse, pois o identificado D1 tem como preocupação central analisar a evolução das habilidades matemáticas, após as intervenções psicopedagógicas em crianças com indícios de DD, enquanto o D2 propõem-se a verificar a influência do lúdico na autoestima e na autoimagem em crianças com DD, após as intervenções educativas. Todavia, elas convergem em relação à preocupação de propiciar aos estudantes com DD, intervenções psicopedagógicas por meio do lúdico.

No que tange aos autores selecionados, para fundamentar a concepção sobre DD, destaca-se: Bastos (2006) (1); Benton (1987) (1); Butterworth (2005) (1); Dehaene (1995) (1); Fonseca (1995) (2); García (1998) (2); Haase, Costa, Michelli, Oliveira, Wood (2011) (1), Kosc (1974) (2), Lara (2004) (2); Rotta (2006) (1); Vieira (2004) (2). Sendo assim, a fundamentação teórica apresentada nas dissertações, convergem quanto a sua organização, pois apresentam questões relevantes sobre Neurociências e Educação e DD. 
Referente ao problema de pesquisa menciona-se: Como intervenções psicopedagógicas repercutiram na evolução do desenvolvimento das habilidades matemáticas envolvidas na DD em crianças com indícios desse transtorno? (1) e Como o lúdico pode influenciar nos níveis de autoestima e de autoimagem em crianças com DD? (1). Quanto ao interesse de pesquisa, tem-se: preocupação com a problemática acerca das DAM e a importância do trabalho educativo que contemple os diferentes estilos de aprendizagem, possibilitando que as crianças com DAM possam aprender (2). Verificou-se que as dissertações convergem em relação ao interesse de pesquisa, ambas preocupamse com os problemas na aprendizagem da Matemática e tem como intenção conscientizarem os profissionais da educação sobre a necessidade de possibilitar meios para que os estudantes com DD possam aprender.

Em se tratando do contexto de pesquisa, os testes e intervenções realizados com os estudantes foram desenvolvidos no laboratório de aprendizagem (2).

Quanto as principais metodologias, menciona-se o método de pesquisa à abordagem qualitativa e quantitativa (2), e em se tratando do tipo de delineamento, optou-se pelo delineamento experimental (1) e pelo delineamento de estudo de caso (1). Quanto às principais metodologias, ambas as dissertações convergem quanto utilização do método de pesquisa de abordagem qualitativa e quantitativa. Todavia elas diferem-se em relação ao tipo de delineamento de pesquisa, enquanto D1 utilizou-se do delineamento experimental, D2 usou-se do delineamento de estudo de caso.

E no que concerne aos principais resultados, as dissertações convergem, pois verificou-se que as intervenções psicopedagógicas desenvolvidas com os participantes, por meio do lúdico, possibilitaram avanços no desempenho em Matemática (2).

No que se refere ao identificador D1, suas contribuições se relacionam a eficácia da utilização de jogos e materiais manipulativos para o tratamento de crianças com indícios de DD, concluindo assim que as habilidades matemáticas em defasagem podem ser reabilitadas, potencializando as existentes. Já o identificador D2, salienta a necessidade da inclusão no ambiente escolar, na intenção de potencializar o desenvolvimento integral dos estudantes, impedindo a repetência, o fracasso, a evasão e, consequentemente, a exclusão social. As dissertações diferem-se em suas contribuições, pois o identificador D1, propõe a utilização de jogos e materiais manipulativos para o tratamento de crianças com indícios de DD, baseando-se nas categorias definidas por Kosc (1974), possibilitando reabilitar e 
potencializar as habilidades em defasagens. Enquanto, a autora do identificador D2, destaca a importância do lúdico nas intervenções no âmbito escolar, em específico nos laboratórios de aprendizagem, auxiliando nas dificuldades, na autoestima, na autoimagem, motivando assim, os estudantes em sua aprendizagem.

\section{Artigos selecionados no Google Acadêmico}

$\mathrm{Na}$ intenção de apresentar os artigos selecionados para leitura integral, organizou-se o Quadro 7, constando código, ano de publicação, título e autor(es).

Quadro 7 - Artigos selecionados para leitura integral e análise

\begin{tabular}{|c|c|c|c|}
\hline CÓDIGO & ANO & TiTULO & AUTOR (ES) \\
\hline A1 ${ }^{2}$ & 2016 & $\begin{array}{c}\text { Discalculia: a utilização de } \\
\text { estratégias de intervenção } \\
\text { baseadas no lúdico }\end{array}$ & $\begin{array}{c}\text { Edneia Felix de Matos; } \\
\text { Daniela Miranda Fernandes } \\
\text { Santos }\end{array}$ \\
\hline A2 & 2011 & $\begin{array}{c}\text { Discalculia: conhecer para } \\
\text { incluir }\end{array}$ & $\begin{array}{c}\text { Jussara Bernardes; Claus } \\
\text { Dieter Stobaus }\end{array}$ \\
\hline
\end{tabular}

Fonte: Elaborado pelas autoras a partir dos dados disponíveis no Google Acadêmico.

Considerando a leitura minuciosa dos artigos, apresenta-se uma síntese detalhada dos aspectos relevantes, no Quadro 8, considerando os seguintes aspectos: título; autor(es); ano de publicação; palavras-chave; objetivo geral; autores selecionados para definir DD; contribuições para o avanço do tema na área do ensino de Matemática e principais referências.

Quadro 8 - Artigos selecionados

\begin{tabular}{|c|c|}
\hline IDENTIFICADOR & A1 \\
\hline \multicolumn{2}{|c|}{$\begin{array}{c}\text { Discalculia: a utilização de estratégias de intervenção baseadas no lúdico. Autores: } \\
\text { Edneia Felix de Matos; Daniela Miranda Fernandes Santos }\end{array}$} \\
\hline ANO & 2016 \\
\hline PALAVRAS-CHAVE & Aprendizagem. Matemática. Discalculia. \\
\hline OBJETIVO GERAL & $\begin{array}{l}\text { Discutir o conceito da DD, suas características, formas de manifestação } \\
\text { entre as crianças e explicitar algumas estratégias de intervenções } \\
\text { baseadas no lúdico, que podem ser utilizadas por professores em sala } \\
\text { de aula para trabalhar com estudantes com esse transtorno. }\end{array}$ \\
\hline $\begin{array}{l}\text { AUTORES UTILIZADOS PARA } \\
\text { DEFINIR DD }\end{array}$ & $\begin{array}{l}\text { Bastos (2006); Bernardi (2014); Campos (2014); Garcia (1998); Kosc } \\
\text { (1974); Lara (2004); Vieira (2011); Vieira (2004). }\end{array}$ \\
\hline $\begin{array}{l}\text { INTERESSES DE PESQUISA } \\
\text { DOS AUTORES }\end{array}$ & $\begin{array}{l}\text { A convivência com estudantes com DD suscitou questionamentos e } \\
\text { reflexões a respeito de um trabalho educativo que contemple os } \\
\text { diferentes estilos de aprendizagem, possibilitando que as crianças } \\
\text { possam aprender. }\end{array}$ \\
\hline
\end{tabular}

\footnotetext{
${ }^{2}$ Para representar cada produção utilizou-se An (A1, A2, ...) para Artigos.
} 


\begin{tabular}{|c|c|}
\hline CONTRIBUIÇÕES & $\begin{array}{l}\text { Por meio da análise de A1 verifica-se que a intensão dos autores foi } \\
\text { possibilitar a ampliação das discussões sobre a DD, pois existem poucas } \\
\text { produções cientificas que contemplam esse tema. }\end{array}$ \\
\hline REFERÊNCIAS & $\begin{array}{l}\text { BASTOS, J. A. Discalculia: transtorno específico da habilidade em } \\
\text { matemática. Porto Alegre: Artes Médicas, } 2006 \text {. } \\
\text { BERNARDI, J. Discalculia: O que é? Como intervir? Jundiaí, Paco } \\
\text { Editora, 2014. } \\
\text { CAMPOS, A. M. A. de. Discalculia: superando as dificuldades de } \\
\text { aprender Matemática. Rio de Janeiro: Wak Editora, } 2014 \text {. } \\
\text { GARCIA, J. N. Manual de dificuldades de aprendizagem: linguagem, } \\
\text { leitura, escrita e matemática. Porto Alegre: Artes Médicas, } 1998 \text {. } \\
\text { KOSC, L. Developmental dyscalculia. Journal of Learning Disabilities, } \\
\text { v. } 7, \text { p. 164-177, } 1974 . \\
\text { LARA, I. C. M. Ensino inadequado de matemática. Revista Ciências e } \\
\text { Letras, n. 35, p.137-152, 2004. } \\
\text { VIEIRA, C. de F.; ZAMPA, R. L. G. A Geometria na Matemática das } \\
\text { Séries Iniciais do Ensino Fundamental. Revista Matemática da UFOP, } \\
\text { v. } 1,2011.64 \\
\text { VIEIRA, E. Transtornos na aprendizagem da matemática: número e } \\
\text { discalculia. Revista Ciências e Letras, n. 35, p. 109-119, 2004. }\end{array}$ \\
\hline \multirow{2}{*}{\multicolumn{2}{|c|}{\begin{tabular}{c|c} 
IDENTIFICADOR & A2 \\
Discalculia: conhecer para incluir. Autores: Jussara Bernardi; Claus Dieter Stobaus \\
\end{tabular}}} \\
\hline & \\
\hline ANO & 2011 \\
\hline PALAVRA-CHAVE & Discalculia. Autoestima e autoimagem. Lúdico. \\
\hline OBJETIVO GERAL & $\begin{array}{l}\text { Verificar a influência do lúdico na autoestima e autoimagem de } \\
\text { participantes com Discalculia. }\end{array}$ \\
\hline $\begin{array}{l}\text { AUTORES UTILIZADOS PARA } \\
\text { DEFINIR DD }\end{array}$ & Fonseca (1995); Garcia (1998); Kosc (1974); Vieira (2004). \\
\hline $\begin{array}{l}\text { INTERESSES DE PESQUISA } \\
\text { DOS AUTORES }\end{array}$ & $\begin{array}{l}\text { De acordo com a autora, consciente dos desafios enfrentados pela } \\
\text { escola e em se tratando dos estudantes com DAM, os quais acabam } \\
\text { apresentando pouca ou nenhuma motivação, diante dos conteúdos e } \\
\text { sabendo da pouca expectativa dos educadores em relação a esses } \\
\text { estudantes, acabam por não desenvolver uma intervenção adequada. } \\
\text { Diante desse quadro, os autores tiveram como interesse propor } \\
\text { intervenções psicopedadagógicas baseadas no lúdico para atender } \\
\text { estudantes com DD. }\end{array}$ \\
\hline CONTRIBUIÇÕES & $\begin{array}{l}\text { Destaca-se a necessidade do olhar educacional inclusivo, com vistas a } \\
\text { atender à diversidade dos estudantes, compreendendo a escola como } \\
\text { um ambiente preparado para atender as dificuldades dos estudantes, } \\
\text { promovendo o seu desenvolvimento integral. Os autores propõe a } \\
\text { utilização de constantes intervenções, podendo assim, auxiliar em suas } \\
\text { dificuldades, melhorar a autoestima, a autoimagem e a motivação para } \\
\text { aprender. }\end{array}$ \\
\hline PRINCIPAIS REFERÊNCIAS & $\begin{array}{l}\text { FONSECA, Vítor. Introdução às dificuldades de aprendizagem. 2. ed. } \\
\text { Porto Alegre: Artes Médicas, } 1995 . \\
\text { GARCIA, José N. Manual de dificuldades de aprendizagem: } \\
\text { linguagem, leitura, escrita e matemática. Porto Alegre: Artes Médicas, } \\
\text { 1998. } \\
\text { KOSC, Ladislav. Developmental dyscalculia. Journal of Learning } \\
\text { Disabilities, v. 7, p. } 164177,1974 \text {. } \\
\text { VIEIRA, Elaine. Transtornos na aprendizagem da matemática: número } \\
\text { e discalculia. Revista Ciências e Letras, n. 35, p. 109-119, } 2004 \text {. }\end{array}$ \\
\hline
\end{tabular}

Fonte: Elaborado pelas autoras.

Tendo em vista a análise dos artigos selecionados, observa-se que a preocupação dos autores com o tema DD, está relacionada à suas experiências enquanto educadores e 
sobre a necessidade dos profissionais da área da educação compreenderem as principais causas e possibilitar perspectivas de como intervir pedagogicamente com esses estudantes.

Referente ao objetivo geral, foi possível observar que os artigos convergem quanto à importância da utilização da ludicidade nas intervenções para estudantes com DD.

Convém mencionar, que os artigos baseiam-se nos autores: Garcia (1998); Kosc (1974); Vieira (2004), para definir DD. Convém mencionar, que a fundamentação teórica dos artigos converge, quanto aos dados trazidos sobre DD.

Em se tratando das contribuições, percebe-se a preocupação dos autores em proporcionar a discussão da temática DD entre os professores (1) e a necessidade da inclusão nas escolas, proporcionando atender todos os estudantes visando o seu desenvolvimento integral, por meio de intervenções baseadas no lúdico (1). Em se tratando das contribuições, percebe-se que os artigos diferem-se, pois o identificador A1, por meio de uma RSL, preocupa-se em ampliar as discussões sobre a DD, enquanto o identificador A2 apresenta os resultados advindos da dissertação "Alunos com DD: o resgate da autoestima e da autoimagem através do lúdico", a qual foi analisada anteriormente.

\section{Considerações finais}

Este estudo teve como objetivo realizar uma RSL, analisando dissertações e artigos, na intenção de verificar a existência de propostas psicopedagógicas para potencializar e reabilitar as habilidades matemáticas em defasagem na DD, sem período de tempo estipulado, a partir da consulta ao banco de dados disponibilizados pela BDTD e Google Acadêmico.

Considerando a análise realizada referente às dissertações e artigos, sobre intervenções para estudantes do Ensino Fundamental com DD, na intenção de responder à questão que guiou esta pesquisa, verificou-se que ainda há poucas pesquisas relacionadas à DD no Brasil, dificultando o processo de avaliação e intervenção psicopedagógica. Isso evidencia a necessidade de que pesquisadores se dediquem ao desenvolvimento de pesquisas que tenham como objetivo a elaboração de instrumentos de avaliação capazes de propiciarem com precisão que tipo de habilidade está em defasagem em cada uma das categorias de DD definidas por Kosc (1974), pois os instrumentos padronizados de avaliação existentes para utilização de professores e psicopedagogos tem como objetivo avaliar as DAM e não a DD. 
A escassez de literatura e a falta de instrumentos padronizados para a avaliação dificulta o processo de intervenção do psicopedagogo, impedindo-o muitas vezes, de realizar intervenções que de fato reabilitem as habilidades debilitadas.

Convém mencionar que se as produções e os artigos selecionados fossem outros, outras considerações certamente seriam tecidas. Todavia, as conclusões parciais que aqui se evidenciam traz à tona a relevância de estudos voltados à questão de intervenções psicopedagógicas que possam ser desenvolvidas com estudantes com DD. Preocupações que já vêm sendo abordadas pelo Grupo de Pesquisas e Estudos sobre Discalculia ${ }^{3}$.

\section{Referências}

AVILA, L. A. B. Avaliação e intervenções psicopedagógicas em crianças com indícios de Discalculia. Porto Alegre, 2017. Dissertação (Mestrado) - Pontifícia Universidade Católica do Rio Grande do Sul, 2017.

AVILA, L. A. B; LARA, I. C. M. Discalculia: Um mapeamento de artigos brasileiros. Abakós. Minas Gerais, v.6, p. 35-56, 2017.

GERSTMANN, J. Some notes on the Gerstmann syndrome. Neurology, p. 866-869, 1957.

KOSC, L. Developmentol Dyscalculia. Journal of Learning Disabilities can be found at, v. 7, n. 3, p.163-177, marc. 1974.

MYKLEBUST, H. R; JOHNSON, D. J. Dyslexia in Chindren. Exceptional Children, p. 14-25, 1962.

PICKERING, C.; BYRNE, J. The benefits of publishing systematic quantitative literature reviews for $\mathrm{PhD}$ candidates and other early career researchers. Higher Education Research and Development, v. 33, n. 3, 534-548, 2014.

\section{Correspondência}

Lanúzia Almeida Brum Avila - Universidade Católica do Rio Grande do Sul. Av. Ipiranga, 6681 - Partenon. CEP: 90619-900. Porto Alegre, Rio Grande do Sul, Brasil.

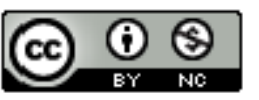

This work is licensed under a Creative Commons Attribution-NonCommercial 4.0 International (CC BY-NC 4.0)

\footnotetext{
${ }^{3}$ Grupo de Estudos e Pesquisas sobre Discalculia - PUCRS, coordenação Dra. Isabel Cristina Machado de Lara.
} 